AUTOPHAGY

\section{Deubiquitinating p53}

Cell 147, 223-234 (2011)

Vsp34, a class III phosphoinositide-3kinase (PI3K), assembles into multiprotein complexes that include Beclin 1 to promote autophagy, a cellular process for the degradation of proteins and organelles. Liu et al. performed an image-based screen followed by extensive structure-activity relationship studies to find spautin- 1 , a potent inhibitor of autophagy in mammalian cells. Spautin-1 treatment led to reduced amounts of Beclin 1 and other components of Vsp34 complexes by promoting the ubiquitination of Beclin1, leading to the hypothesis that spautin-1 inhibits deubiquitinating enzymes (DUBs). To test this, the authors screened siRNAs targeting DUBs; knockdown of USP10 or USP13 resulted in decreased Beclin1 expression and inhibited autophagy. In contrast, overexpression of USP10 or USP13 reduced amounts of ubiquitinated Beclin1. Further investigations revealed that Beclin1 directly interacted with USP13, promoting its DUB activity, and indirectly promoted USP10 activity. In cells treated with spautin-1 and in mice heterozygous for the Beclin1 gene, the authors found that amounts of p53, a known substrate for USP10, were decreased. Together, these data support a model in which class III PI3K components, including Beclin1, regulate $\mathrm{p} 53$ levels via direct interaction with DUBs and provide a potential mechanistic explanation for Beclin1's reported tumor-suppressor activity.

LIPIDS

\section{Coming together}

Cell Metab. 14, 504-515 (2011)

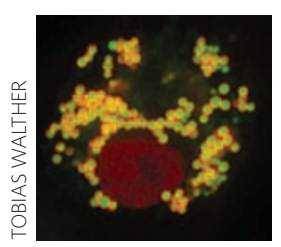

Lipid droplets (LD) store neutral sterol esters and triglyceride (TG) lipids in their cores, which are limited by a phospholipid monolayer. LD size fluctuates considerably in response to TG storage needs. More is known about how core lipids are generated during lipid flux than about how membrane phospholipids are generated, though knockdown of mammalian CCT enzymes that catalyze the rate-limiting step of phosphatidylcholine (PC) biosynthesis causes fusion of smaller LDs to form enlarged LDs. Krahmer et al. now confirm this result in Drosophila melanogaster cells and further show that the phenotype is reversed by addition of PC-containing liposomes. Through experiments using artificial LDs of different phospholipid compositions, the authors also found that PC acts as a surfactant that stabilizes LDs and prevents their coalescence and subsequent fusion. Among the PC biosynthetic enzymes, only CCT localized to LDs, suggesting that it has a regulatory purpose. Indeed, the authors show that when the amount of TG increases, the concentration of PC on LDs initially drops, leading to the targeting of CCT to
TRANSLATION

\section{Protein polyglots}

Nature $477,490-494$ (2011)

Polyamines such as spermine are polycationic molecules with multiple cellular functions. In eukaryotes, polyamine biosynthesis involves the conversion of ornithine to putrescine by ornithine decarboxylase (ODC). The biosynthetic pathway is regulated by modulation of ODC concentrations in a peculiar process in which an ODC 'antizyme' (OAZ) disrupts ODC dimerization, thereby targeting it for degradation. Production of full-length OAZ1 protein in Saccharomyces cerevisiae requires +1 ribosomal frameshifting (RFS) during translation, a process that is enhanced by elevated polyamine concentrations. Kurian et al. now show that polyamines directly regulate antizyme expression by binding to the nascent OAZ1 polypeptide while it is still on the ribosome. A detailed analysis of OAZ1 mRNA revealed that sequences $5^{\prime}$ and $3^{\prime}$ to the RFS site were required to negatively regulate OAZ1 expression, but surprisingly, the regulatory element resides in the emerging OAZ1 polypeptide and not in the coding mRNA. An N-terminal polypeptide domain of OAZ1 negatively controls RFS whereas a C-terminal domain prevents completion of OAZ1 translation under low-polyamine conditions. Biochemical data showing direct polyamine binding to OAZ1 led the authors to a regulatory model explaining how elevated concentrations of polyamines enhance OAZ1 translation, leading to ODC turnover and downregulation of polyamine biosynthesis. This example of metabolite sensing by nascent polypeptides during translation may represent a new pathway for the regulation of gene expression in cells. the expanding LD surface, an event that activates this enzyme and thus increases PC synthesis. Once PC concentrations stabilize, CCT no longer binds to LDs and-owing to its nuclear localization sequence-trafficks to the nucleus. The results suggest that CCT regulation by $\mathrm{LD}$ binding is most likely part of a dynamic, homeostatic mechanism for PC synthesis. $M B$

BIOSYNTHESIS

\section{A dehydratase in hiding}

ChemBioChem., published online 27 September 2011, doi:10.1002/cbic.201100474

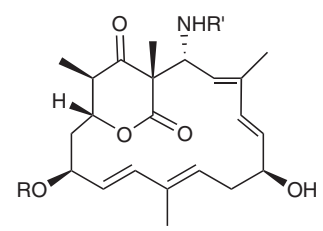

The lankacidins are macrocyclic polyketides that contain four carboncarbon double bonds and are synthesized by hybrid machinery containing both nonribosomal peptide synthetase and polyketide synthase modules. Olefin formation is thought to be catalyzed by a trans-acting dehydratase, $\mathrm{LkcB}$, in tandem with the LkcC subunit, which is responsible for selecting incoming building blocks to lengthen the molecular chain. However, the current biosynthetic proposal, in which the LkcB-LkcC pair function iteratively to complete five steps of the pathway, does not agree with the substrate specificity of the $\mathrm{LkcC}$ subunit. In efforts to elucidate the true biosynthetic pathway, Dickschat et al. resequenced the lankacidin-producing Streptomyces rochei var. volubilis ATCC 21250. Analysis of this sequence identified a previously unassigned $\sim 400$-amino-acid segment of LkcC containing both a second dehydratase domain and a putative docking site for a trans-acting acyltransferase. Mutational analysis of both the $\mathrm{LkcB}$ and $\mathrm{LkcC}$ dehydratases confirmed that their function was necessary for lankacidin production, although the lack of any intermediate lankamycin products precluded revision of the biosynthetic proposal. However, the authors do speculate that, in a process akin to the mechanism used by FabA in fatty acid biosynthesis to determine product outcome, chain-length selectivity of the new dehydratase might determine the reaction course, in this case by dictating a switch between iterative cycles through LkcC and modular steps through other lankamicin subunits. 